\title{
Our Experience with Percutaneous and Surgical Tracheotomy in Intubated Critically Ill Patients
}

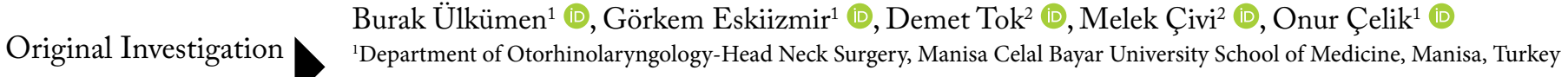 \\ ${ }^{2}$ Department of Anesthesiology and Reanimation, Manisa Celal Bayar University School of Medicine, Manisa, Turkey
}

\section{Abstract}

ORCID IDs of the authors: B.Ü. 0000-0003-1981-5886; G.E. 0000-0002-3125-8288; D.T. $0000-0002-0430-1754$ M.C. $0000-0002-8239-8037$ 0.C. 0000-0001-9188-3467.

Cite this article as: Ülkümen B, Eskiizmir G, Tok D, Çivi M, Çelik 0. Our Experience with Percutaneous and Surgical Iracheotomy in Intubated Critically III Patients. Turk Arch Otorhinolaryngol 2018; 56(4): 199-205.

This study was presented at the $39^{\text {th }}$ Turkish National Congress of Otorhinolaryngology Head and Neck Surgery, November 8-12, 2017, Antalya, Turkey. Corresponding Author: Burak Ülkümen; drburak@gmail.com Received Date: 13.06 .2018 Accepted Date: 12.10 .2018

( ) Copyright 2018 by Official Journal of the Turkish Society of Otorhinolaryngology and Head and Neck Surgery Available online at www.turkarchotolaryngol.net DO1: $10.5152 / t a 0.2018 .3603$
Objective: Open surgical tracheotomy (OST) and percutaneous dilatational tracheotomy (PDT) are commonly used for securing airway in intubated critically ill patients. The purpose of this study was to compare the safety of OST and PDT, particularly in intubated critically ill patients.

Methods: The medical records of intubated critically ill patients who underwent tracheotomy between $\mathrm{Au}^{-}$ gust 2006 and July 2017 were analyzed retrospectively. Minor and major complication rates were compared according to the tracheotomy technique. Preoperative intubation time, postoperative decannulation time, reason for hospitalization, and demographic data, including the Acute Physiology and Chronic Health Evaluation (APACHE II) and Sequential Organ Failure Assessment (SOFA) scores, were evaluated.

Results: A total of 332 cases were enrolled into the study. The minor and major complication rates for both techniques were $27.2 \%, 8.8 \%, 9.7 \%$ and $3.2 \%$, respecti- vely. Minor and major complication rates were higher in the OST group ( $p=0.01, p=0.03$, respectively). The rate of every single complication was also compared on groups' basis. Accidental decannulation $(\mathrm{p}=0.02)$ and pneumothorax $(\mathrm{p}=0.05)$ were found to be significantly frequent in the OST group. There was no impact of the preoperative intubation time on the minor $(\mathrm{p}=0.20)$ and major complication $(\mathrm{p}=0.29)$ rates found. There was no statistically significant difference regarding the postoperative decannulation time $(\mathrm{p}=0.32)$. Also, there was no statistically significant difference between two groups in terms of the APACHE II $(\mathrm{p}=0.69)$ and SOFA ( $p=0.37)$ scores. However, a statistically significant difference between the groups in terms of overall survival was found, in favor of PDT ( $\mathrm{p}<0.001)$.

Conclusion: This study revealed that PDT is safer than OST, particularly in intubated critically ill patients.

Keywords: Tracheotomy, methods, complication, intensive care

\section{Introduction}

Open surgical tracheotomy (OST) is one of the oldest surgical interventions used in the upper airway insufficiency. The oldest known text describing tracheotomy is an ancient sacred Hindu book called the Rig Veda, dating back to 2000 BC. Nevertheless, the tracheotomy statement was first defined by Lorenz Heister in 1739 (1). Currently, common indications for tracheotomy are upper airway insufficiency (UAI) and prolonged intubation (PI) $(1,2)$. In the past, infections of the upper airway (diphtheria, epiglottitis, etc.) causing UAI were the leading indications. But nowadays, PI became the main indication because of current treatment modalities for head and neck infections and common use of mechanical ventilation (3).
Although OST dates back to distant past, it was popularized in the 19th century. On the contrary, percutaneous dilatational tracheotomy (PDT) (4), which was initially described by Ciaglia et al. (5) in 1985, has a quite short history. However, PDT has gained a great interest and has been widely performed, especially in intensive care units (ICU), in the past three decades (6).

Open surgical tracheotomy and PDT have same indications, such as the upper airway obstruction, maxillofacial procedures, weaning from CPAP in respiratory failure/prolonged ventilation, and preventing aspiration in patients with a compromised cough reflex $(7,8)$. Besides, each tracheotomy technique has its own advantages and disadvantages with regard to some factors, such as the tim- 
ing of procedure, size of incision, amount of dissection, and type and rate of complications (1). Concerning safety, type and rate of complications come to the forefront.

The purpose of this study was to find out which tracheotomy technique is safer, especially in critically ill intubated patients in the ICU. For this reason, minor and major complication rates of OST and PDT in intubated critically ill patients were evaluated. We also evaluated the preoperative intubation time (PIT), postoperative decannulation time (PCT), reason for hospitalization (RfH), and demographics including the Acute Physiology and Chronic Health Evaluation (APACHE II) and Sequential Organ Failure Assessment (SOFA) scores for any possible impact on complication rates.

\section{Methods}

This study was approved by the Ethical Committee of Manisa Celal Bayar University (approval protocol number: 09/09/2015.20478486-349). It was designed as a retrospective cohort study. Thus, no written informed consent was obtained from the patients. Power analyses predicted a sample size of 195 patients with a power of $95 \%$ and the effect size of $\alpha 2=0.05$.

\section{Study population}

The files of 423 intubated critically ill patients who underwent OST or PDT from August 2006 to July 2017 were analyzed. Nighty-one cases with malignant neoplasms of the upper aerodigestive tract were excluded. Hence, a total of 332 suitable cases were enrolled into the study.

\section{Complications and influential factors}

Gender, age, RfH, indication for tracheotomy, PIT, PCT, and complications (minor and major) were determined in the first stage of the study. Patients with a PIT of $\leq 7$ days were classified as "early tracheotomy," while patients with a PIT of $>7$ days were classified as "late tracheotomy." The APACHE II, SOFA score, and the rate of expected mortality of each patient were calculated at the beginning of the ICU admission. In addition, the SOFA and Glascow Coma Scale (GCS) scores before the operation were also noted.

Minor complications were categorized as the following: (i) accidental decannulation, (ii) subcutaneous emphysema, (iii) wound infection, (iv) hemorrhage, and (v) keloid formation. Major complications were categorized as (i) accidental decannulation with airway compromise, (ii) pneumothorax necessitating chest tube, (iii) hemorrhage requiring surgery, (iv) tracheomalacia, (v) esophagotracheal fistula, (vi) fistula of the tracheoinnominate artery, and (vii) death caused by tracheotomy (cardiac or respiratory arrest during procedure).

\section{Tracheotomy procedures}

All OST procedures were performed at the operating theater, whereas PDTs were performed at bedside in the ICU. In all patients who underwent OST, after the incision and dissection of the strap muscles, a tracheal flap having an inferior base was prepared between the 2nd and 3rd tracheal ring and favorably sutured to the skin to enable an emergent recannulation in case of accidental decannulation. The PDT was performed by a percutaneous tracheotomy kit (GRIGGS, Portex, Smiths Medical, Ashford, United Kingdom) as described by Griggs et al. (9). A horizontal skin incision was done below the level of the cricoid cartilage. Thereafter, a mosquito clamp was used to separate the strap muscles to palpate the trachea through the incision. Then, the tracheal lumen was penetrated between the 2 nd and 3rd tracheal rings with a needle followed by a guide-wire placement. Finally, gradual dilatation with a tracheal dilating forceps was done, and a tracheotomy cannula was placed into the tracheal lumen. Bronchoscopy was not used routinely during the PDT, except in cases where there was a concern with regard to the place and the level of the needle and/or guide-wire.

\section{Statistical Analysis}

Each individual complication rate and the overall minor and major complication rates of both groups were analyzed. The APACHI II, SOFA score, and GCS of each group were also compared. The data were presented as the mean \pm standard deviation (SD), and the distribution was evaluated by the Shapiro-Wilk test. Data comparison was done by the two-sample $t$ test or Mann-Whitney $U$ test with respect to the results of the normality test (Kolmogorov-Smirnov). The Kaplan-Meier test was used for survival analyses. The log rank (Mantel-Cox) was used for comparison of the overall survival in terms of the tracheotomy technique. Statistical significance was defined as $\mathrm{p}<0.05$. The Statistical Package for the Social Sciences (SPSS) Version 21.0 (IBM Corp.; Armonk, NY, USA) was used for statistical calculations.

\section{Results}

\section{Descriptive statistics}

A total of 332 cases were included in the study. A hundred and fourteen cases (55 females, 59 males; mean age $\pm \mathrm{SD}$, $47.66 \pm 24.09)$ underwent OST at the operating room, whereas the remaining 218 patients (113 females, 105 males; mean age $\pm S D, 58.26 \pm 15.22)$ underwent PDT at bedside. The age distribution demonstrated a statistically significant difference between the PDT and OST groups $(\mathrm{p}<0.001)$.

Two principle indications for tracheotomy were UAI ( $27 \mathrm{pa}^{-}$ tients, 8.2\%) and PI (305 patients, 91.8\%), and the RfH in patients who had tracheotomy due to PI and UAI are demonstrated in Table 1.

At the time of admission to the ICU, the mean APACHE II score of the OST and PDT group was 21.50 \pm 5.95 and $21.75 \pm 6.4$, while the mean SOFA score was $6.21 \pm 1.42$ and $6.09 \pm 1.35$, respectively. Prior to the operation, the mean SOFA score of the OST and PDT group was $6.13 \pm 1.37$ and $6.35 \pm 1.53$, respectively. The percentage of expected mortality for the OST and PDT group was $40.09 \pm 19.01$ and $38.93 \pm 18.32$, respectively. The test of normality values for APACHE II, SOFA scores, and the percentage of expected mortality were calculated by the Shapiro-Wilk test. The distribution of APACHE II, SOFA 
scores and percentage of expected mortality was found to be abnormal $(p<0.05)$ for the initial as well as for the data before the operation. Thus, the Mann-Whitney U test was applied to compare these parameters for both groups. No statistically significant difference in terms of the APACHE II $(p=0.69)$ and SOFA ( $\mathrm{p}=0.37)$ scores was found between the OST and PDT at the time of admission. Again, no statistically significant difference in terms of the SOFA ( $\mathrm{p}=0.19)$ score was found at the time of operation. There was also no statistically significant difference found in terms of the expected mortality on the group basis $(\mathrm{p}=0.60)$.

The mean GCS just prior to surgery for OST and PDT were $9.68 \pm 2.80$ and $9.32 \pm 2.97$, respectively. The distribution of both was found to be abnormal $(\mathrm{p}<0.05)$, thus the Mann-Whitney $\mathrm{U}$ test was applied for comparison. No statistically significant difference in terms of GCS ( $\mathrm{p}=0.28$ ) was found for the tracheotomy technique.

\section{Timing of tracheotomy, preoperative intubation, and PCT}

In the OST and PDT group, the mean PIT was $10.10 \pm 6.33$ days (range 2-42 days) and $12.20 \pm 6.59$ days (range 1-46 days). The distribution of patients according to the timing of tracheotomy was 86 "early tracheotomies" (45 PDT and 41 OST) and 246 "late tracheotomies" (173 PDT and 73 OST). Normality values for PIT and PCT was calculated by the Shapiro-Wilk test. The distribution of both was found to be abnormal $(\mathrm{p}<0.05)$. Thus, the Mann-Whitney $U$ test was applied to compare these parameters for both groups. No statistically significant difference concerning the PCT $(\mathrm{p}=0.32)$ between the OST and PDT groups was determined. The mean duration of PCT was 170.27 days for PDT and 159.29 days for OST. The PIT was significantly higher in the PDT group when compared with the OST group $(\mathrm{p}<0.001)$.

\section{Minor and major complications}

The minor complication rates for the OST and PDT group were $27.2 \%$ and $8.8 \%$, whereas the major complication rates were $9.7 \%$ and $3.2 \%$, respectively. Minor and major complication rates were significantly higher in the OST group when compared with the PDT group ( $\mathrm{p}=0.01, \mathrm{p}=0.03$, respectively). However, a vast majority of the minor and major complications except accidental decannulation and pneumothorax were not found to be significantly high when analyzed individually. There was no predominance of any major complication on the group basis, but hemorrhage was the most frequent minor complication in both groups (Table 2). In addition, no statistically significant difference was determined between early and late tracheotomies in terms of the overall minor $(\mathrm{p}=0.29)$ and major $(\mathrm{p}=0.20)$ complication rates. Moreover, the individual comparison of each complication also did not demonstrate any statistically significant difference in terms of timing (Table 3).

Two esophagotracheal fistulae cases had occurred in each group (Table 3). In the PDT group, the first case was a 78-year-old woman with chronic obstructive pulmonary disease having cough associated with eating, and saliva and gastric content ex- cretion from the tracheotomy site. These symptoms appeared one month after the PDT. Diagnosis of an esophagotracheal fistula was confirmed on the 10th day of symptoms. The second case was a 69-year-old woman with head injury, which manifested relevant symptoms two months after PDT, and the diagnosis was confirmed in two weeks. In the OST group; the first case was a 27 -year-old man with maxillofacial trauma having relevant symptoms on the 23rd day of PDT. The definitive diagnosis was confirmed in two weeks. The second case in the OST group was a 50-year-old woman with a cerebrovascular disorder having symptoms the third week following OST, and the diagnosis was confirmed in 10 days. In all four cases, the diagnosis was confirmed by $3 \mathrm{D}$ computed tomography and bronchoscopy. Tracheomalacia was seen only in a 35-year-old man with a head injury, who underwent PDT. In this case, inspiratory stridor and dyspnea were seen following decannulation after six months of cannulation. The diagnosis was confirmed by bronchoscopy.

\section{Overall survival and duration of ICU hospitalization}

In the OST and PDT group, the observation time was $241.83 \pm 168.23$ (range, $1-848$ days) and $375.01 \pm 187.49$ (range, 38-850 days), respectively. The overall survival rates for OST and PDT were $71.1 \%$ and $79.4 \%$, respectively. The log rank (Mantel-Cox) test was used for the comparison of the overall survival rate between the groups, and it was found to be significantly high in the PDT group (Figure 1) $(\mathrm{p}<0.001)$. The distribution of duration of ICU hospitalization was abnormal $(\mathrm{p}<0.05)$, so Mann-Whitney $U$ test was used for comparison between groups. The mean duration of ICU hospitalization was 172.60 days for the patients who underwent PDT and 154.84 days for OST. There was no statistically significant difference between OST and PDT in terms of the ICU stay ( $p=0.11)$.

\section{Discussion}

In concordance with the literature, the majority of our tracheotomy cases consisted of patients with PI (91.8\%). Cardiovascular diseases and cerebrovascular disorders were the leading $\mathrm{RfH}$ in both the OST and PDT groups (Table 1). This is also concordant with similar studies $(10,11)$.

There have been many researchers evaluating the safety of tracheotomy techniques; however, there is still no consensus on the overall superiority of any technique $(4,7,8,11,12)$. Indeed, OST and PDT have some advantages and disadvantages in different aspects, such as the timing of procedure, size of incision, amount of dissection, and complication rates. As a matter of fact, the complication rate is the most important factor in deciding the type of tracheotomy technique. In this study, the overall complication rate was $17.4 \%$ (58/332) irrespective of the tracheotomy technique. Both the minor and major complication rates were significantly higher in the OST group ( $\mathrm{p}=0.01$ and $\mathrm{p}=0.03$ ). It is noteworthy that this difference may be related to the decision-making process concerning the type of tracheotomy technique, which is the blind side of this study like other retrospective studies. In our institution, decision on the tracheotomy technique for intubated patients was taken by the intensivists who roughly consider some patient-related risk factors, such as 
Ülkümen et al.

202 Percutaneous and Surgical Tracheotomy

Table 1. Indications and reasons for hospitalization according to the tracheotomy technique

\begin{tabular}{|c|c|c|c|}
\hline Reason for Hospitalization & OST n (\%) & PDT n (\%) & (Total) n (\%) \\
\hline \multicolumn{4}{|l|}{ Prolonged intubation } \\
\hline Cerebrovascular disorder & $31(9.3)$ & $94(28.3)$ & $125(37.6)$ \\
\hline Cardiovascular disease & $19(5.7)$ & $43(12.9)$ & $62(18.6)$ \\
\hline Pneumonia/exacerbation of COPD & $18(5.4)$ & $35(10.5)$ & $53(15.9)$ \\
\hline Other neurological diseases & $16(4.8)$ & $33(9.9)$ & $49(14.7)$ \\
\hline Trauma and/or intoxication & $3(0.9)$ & $6(1.8)$ & $9(2.7)$ \\
\hline Major surgery & $2(0.6)$ & $5(1.5)$ & $7(2.1)$ \\
\hline Total & $89(26.7)$ & $216(64.9)$ & $305(91.6)$ \\
\hline \multicolumn{4}{|l|}{ Upper airway insufficiency } \\
\hline Maxillofacial trauma & $14(4.2)$ & $2(0.6)$ & $16(4.8)$ \\
\hline Laryngotracheal trauma & $3(0.9)$ & - & $3(0.9)$ \\
\hline Bilateral choanal atresia & $3(0.9)$ & - & $3(0.9)$ \\
\hline Deep neck infection & $3(0.9)$ & - & $3(0.9)$ \\
\hline Congenital laryngeal anomaly & $1(0.3)$ & - & $1(0.3)$ \\
\hline Angioedema & $1(0.3)$ & - & $1(0.3)$ \\
\hline Total & $25(7.5)$ & $2(0.6)$ & $27(8.1)$ \\
\hline Overall & $114(34.3)$ & $218(65.7)$ & $332(100)$ \\
\hline
\end{tabular}

OST: open surgical tracheotomy; PDT: percutaneous dilatational tracheotomy; COPD: chronic obstructive pulmonary disease

*Percentages are given with respect to overall population (332)

Table 2. Complication rates according to the tracheotomy technique

\begin{tabular}{|c|c|c|c|}
\hline Type of Complication & $\operatorname{OST} n(\%)^{*}$ & $\operatorname{PDT} n(\%)^{*}$ & $\mathrm{p}$ \\
\hline \multicolumn{4}{|l|}{ Minor } \\
\hline Hemorrhage & $10(8.8)$ & $8(3.7)$ & 0.35 \\
\hline Subcutaneous emphysema & $7(6.1)$ & $3(1.4)$ & 0.16 \\
\hline Keloid formation & $1(0.9)$ & - & 0.17 \\
\hline Accidental decannulation & $7(6.1)$ & $3(1.4)$ & 0.02 \\
\hline Wound infection & $6(5.3)$ & $5(2.3)$ & 0.15 \\
\hline Total & $31(27.2)$ & $19(8.8)$ & 0.01 \\
\hline \multicolumn{4}{|l|}{ Major } \\
\hline Major hemorrhage & $2(1.8)$ & $2(0.9)$ & 0.51 \\
\hline Decannulation leading airway failure & $2(1.8)$ & $1(0.5)$ & 0.24 \\
\hline Pneumothorax requiring chest tube & $2(1.8)$ & - & 0.05 \\
\hline Tracheomalacia & - & $1(0.5)$ & 0.47 \\
\hline Esophagotracheal fistula & $2(1.8)$ & $2(0.9)$ & 0.24 \\
\hline Other life-threatening events & $1(0.9)$ & - & 0.17 \\
\hline Death due to tracheotomy & $2(1.8)$ & $1(0.5)$ & 0.24 \\
\hline Total & $11(9.7)$ & $7(3.2)$ & 0.03 \\
\hline
\end{tabular}

OST: open surgical tracheotomy; PDT: percutaneous dilatational tracheotomy

*Percentages are given according to subgroups

obesity, short neck, goiter, etc. Thus, this might have led to a patient selection bias. But, concerning the relevant literature, we did not find any previous study showing a relationship between these patient-related risk factors and complication rates for any tracheotomy technique, particularly in intubated patients. Nevertheless, to overcome these potentially confounding factors, we also evaluated the APACHE II and SOFA scores, which are known to be the predictors of the prognosis and overall survival. But, we did not find any statistically significant difference between these scores on the group basis. On the other hand, survival analyses revealed a significantly important difference in favor of PDT (Figure 1). 
Table 3. The comparison of complication rates according to timing of tracheotomy

\begin{tabular}{|c|c|c|c|}
\hline Timing of Tracheotomy & Early Tracheotomy n (\%)* & Late Tracheotomy n (\%)* & $\mathrm{p}$ \\
\hline \multicolumn{4}{|l|}{ Minor } \\
\hline Hemorrhage & $6(6.9)$ & $12(4.9)$ & 0.46 \\
\hline Subcutaneous emphysema & $2(2.3)$ & $8(3.3)$ & 0.67 \\
\hline Keloid formation & - & $1(0.4)$ & 0.55 \\
\hline Decannulation & $4(4.7)$ & $6(2.4)$ & 0.30 \\
\hline \multicolumn{4}{|l|}{ Wound infection } \\
\hline & $3(3.5)$ & $8(3.3)$ & 0.92 \\
\hline Total & $15(17.4)$ & $35(14.3)$ & 0.29 \\
\hline \multicolumn{4}{|l|}{ Major } \\
\hline Major Hemorrhage & $2(2.3)$ & $2(0.8)$ & 0.27 \\
\hline Accidental decannulation & - & $3(1.2)$ & 0.30 \\
\hline Pneumothorax & $1(1.2)$ & $1(0.4)$ & 0.44 \\
\hline Esophagotracheal fistula & $1(1.2)$ & $2(0.8)$ & 0.77 \\
\hline Tracheomalacia & $1(1.2)$ & - & 0.09 \\
\hline Other life-threatening events & - & $1(0.4)$ & 0.55 \\
\hline Death & $1(1.2)$ & $2(0.8)$ & 0.77 \\
\hline Total & $6(7.1)$ & $11(4.4)$ & 0.20 \\
\hline
\end{tabular}

*Percentages are presented on group basis

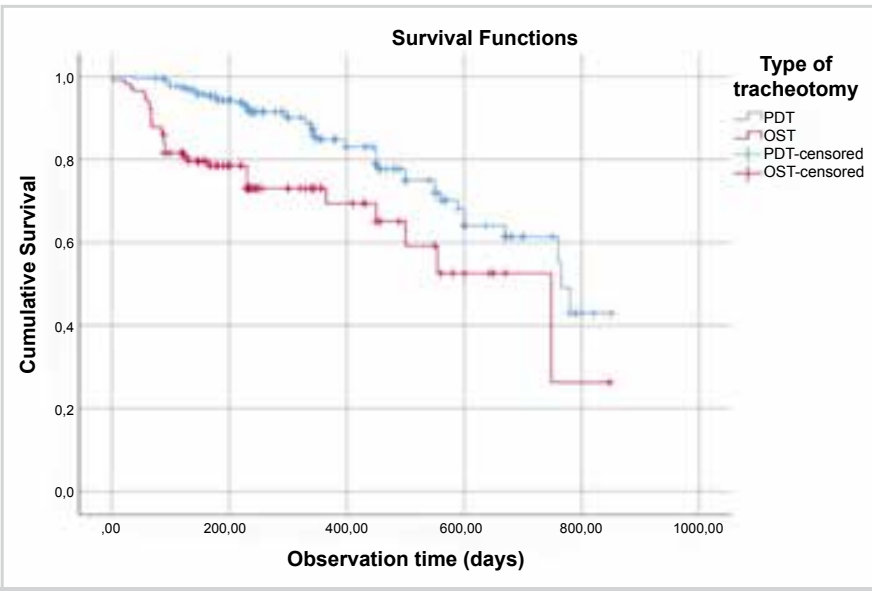

Figure 1. Kaplan-Meier curves of duration of overall survival. PDT group showed statistically significant better overall survival when compared with OST group

Some researchers classify the complications with respect to the "time of tracheotomy" as short term and long term. For instance, Gysin et al. (13) reported an increased incidence of short-term complications along with decreased long-term complications for PDT. On the contrary, we did not classify the complications with respect to the time of tracheotomy, because, to make an implication about the safety of any tracheotomy technique, the ultimate complication rates should be considered rather than the timing of procedure. In this study, along with complication rates as a whole, all specific complications were also analyzed independently. As a result, a statistically significant difference was only detected for minor decannulation (OST, 6.1\%, and PDT, $1.4 \% ; \mathrm{p}=0.02$ ) and pneumothorax (OST, 1.8\%, and PDT, 0\%; $\mathrm{p}=0.05$ ) (Table 2). In previous studies, the incidence of accidental decannulation and pneumothorax was reported to range between $0.5 \%$ and $15 \%(8,2,14)$ and $0 \%$ and $17 \%(15-18)$, respectively. Halum et al. (2) reported the incidence of pneumothorax as $0.2 \%$ in their multi-institutional study; however, they did not give any information concerning the impact of the tracheotomy technique. Delaney et al. (15) reported that PDT was superior to OST in terms of the wound infection and overall mortality, and they suggested that PDT should be considered the technique of choice for critically ill patients. Pappas et al. (18), in their meta-analyses, found no consistent evidence in favor of any technique.

Tracheoinnominate artery fistulae were not seen in any of our cases. Documented risk factors for this lethal and rare complication are utilization of cannulas with a high-pressure cuff, direct trauma by the cannula due to an extreme head tilt, and low surgical level (19). The lack of this complication in the current research may be due to the selection of cannulas with high-volume low-pressure cuffs (Cuffed Flex D.I.C Tracheostomy Tubes, Smiths Medical, Ashford, United Kingdom), which have been used in our institution as previously reported (1).

In the literature, the relationship between the timing of tracheotomy and complications in intubated critically ill patients was reported only for PDT. Möller et al. (20) and Flaatten et al. (21) evaluated the role of PIT on the incidence of particular complications and long-term outcome in patients who underwent tracheotomy at the ICU. However, they did not compare them separately as OST and PDT. Unfortunately, any definition of early/late tracheotomy was debatable. However, a vast 
majority of authors accepted a cut-off date on the 7th day of intubation (22-24); therefore, we also postulated the yardstick as the 7th day. In this study, the OST and PDT groups were compared in terms of PIT for the first time in literature. The PIT was significantly lower in the OST group. This finding might have been the underlying reason for the statistically significant difference of complication rates between PDT and OST groups. But, when we compared the complication rates according to the timing of tracheotomy, we did not find any statistically significant difference (Table 3). However, considering the relevant literature, early tracheotomy is correlated with an earlier ICU discharge, shorter duration of mechanical ventilation, and decreased time of overall hospitalization (2325 ). Besides, the frequency of minor and major complications has not been evaluated separately with respect to timing of tracheotomy in the previous studies. In this study, we revealed that both the minor and major complication rates were relatively high in the early tracheotomy group, but this difference was not statistically significant (Table 3). In brief, we found that the timing of tracheotomy had no effect on complication rates, which was contrary to some previous studies $(22,25)$. This fact may be attributable to two reasons: (i) Tubes with conical shaped cuffs were used in whole study population; (ii) the cuff pressure was measured and tuned up every 6 hours in all patients throughout the preoperative period.

\section{Conclusion}

Concerning critically ill intubated patients, minor and major complication rates were found to be significantly higher in OST when compared to PDT. Minor hemorrhage was found to be the most frequent minor complication of both tracheotomy techniques, whereas no predominance of any major complication was found. The risk of accidental decannulation and pneumothorax were significantly higher in the OST group. The timing of tracheotomy did not increase the complication rates in OST and PDT. The APACHE II, SOFA scores, and expected percentage of mortality in both groups were not statistically different, but the overall survival rates in both groups were found to be significantly different in favor of PDT. Considering these findings, PDT seems to be safer than OST, particularly in critically ill intubated patients.

Ethics Committee Approval: Ethics committee approval was received for this study from the Ethics Committee of Manisa Celal Bayar University School of Medicine (09.09.2015/20478486-349).

Informed Consent: Informed consent was not received due to the retrospective nature of the study.

Peer-review: Externally peer-reviewed.

Author Contributions: Concept - B.U., G.E., D.T., M.Ç., O.Ç.; Design - B.U., G.E., D.T., M.Ç., O.Ç.; Supervision - B.U., G.E., D.T., M.Ç., O.Ç.; Resource - D.T., M.Ç.; Materials - D.T., M.Ç.; Data Collection and/or Processing - B.U., G.E., D.T., M.Ç., O.Ç.; Analysis and/or Interpretation - B.U., G.E., D.T., M.Ç., O.Ç; Literature Search - B.U., G.E., D.T., M.Ç., O.Ç; Writing - B.U., G.E., D.T., M.Ç., O.Ç; Critical Reviews - B.U., G.E., D.T., M.Ç., O.Ç.
Conflict of Interest: The authors have no conflicts of interest to declare.

Financial Disclosure: The authors declared that this study has received no financial support.

\section{References}

1. Ulkumen B, Eskiizmir G, Celik O. The safety of open surgical tracheotomy performed by otorhinolaryngology residents. Pak J Med Sci 2018; 34: 600-5. [CrossRef]

2. Halum SL, Ting JY, Plowman EK, Belafsky PC, Harbarger CF, Postma GN, et al. A multi-institutional analysis of tracheotomy complications. Laryngoscope 2012; 122: 38-45. [CrossRef]

3. Slutsky AS. History of mechanical ventilation from Vesalius to ventilator-induced lung injury. Am J Respir Crit Care Med 2015; 191: 1106-15. [CrossRef]

4. Johnson-Obaseki S, Veljkovic A, Javidnia H. Complication rates of open surgical versus percutaneous tracheostomy in critically ill patients. Laryngoscope 2016; 126: 2459-67. [CrossRef]

5. Ciaglia P, Firsching R, Syniec C. Elective percutaneous dilatational tracheostomy. A new simple bedside procedure; preliminary report. Chest 1985; 87: 715-9. [CrossRef]

6. Koksal GM, Sayilgan $\mathrm{NC}, \mathrm{Oz} \mathrm{H}$. Percutaneous dilatational tracheostomy. Middle East J Anaesthesiol 2006; 18: 903-10.

7. Dulguerov P, Gysin C, Perneger TV, Chevrolet JC. Percutaneous or surgical tracheostomy: a meta-analyses. Crit Care Med 1999; 27: 1617-25. [CrossRef]

8. Friedman Y, Fildes J, Mizock B, Samuel J, Patel S, Appavu S, et al. Comparison of percutaneous and surgical tracheostomies. Chest 1996; 110: 480-5. [CrossRef]

9. Griggs WM, Worthley LI, Gilligan JE, Thomas PD, Myburg JA. A simple percutaneous tracheostomy technique. Surg Gynecol Obstet 1990; 170: 543-5.

10. Brotfain E, Koyfman L, Frenkel A, Semyonov M, Peiser JG, Hayun-Maman $\mathrm{H}$, et al. Bedside percutaneous tracheostomy versus open surgical tracheostomy in non-ICU patients. Crit Care Res Pract 2014; 2014: 156814. [CrossRef]

11. Freeman BD, Isabella K, Lin N, Buchman TG. A meta-analysis of prospective trials comparing percutaneous and surgical tracheostomy in critically ill patients. Chest 2000; 118: 1412-8. [CrossRef]

12. Oliver ER, Gist A, Gillespie MB. Percutaneous versus surgical tracheotomy: an updated meta-analysis. Laryngoscope 2007; 117: 1570-5. [CrossRef]

13. Gysin C, Dulguerov P, Guyot JP, Perneger TV, Abajo B, Chevrolet JC. Percutaneous versus surgical tracheostomy: a double-blind randomized trial. Ann Surg. 1999; 230: 708-14. [CrossRef]

14. Wease GL, Frikker M, Villalba M, Glover J. Bedside tracheostomy in the intensive care unit. Arch Surg 1996; 131: 552-4. [CrossRef]

15. Delaney A, Bagshaw SM, Nalos M. Percutaneous dilatational tracheostomy versus surgical tracheostomy in critically ill patients: a systematic review and meta-analysis. Crit Care 2006;10: R55. [CrossRef]

16. Tarnoff M, Moncure M, Jones F, Ross S, Goodman M. The value of routine posttracheostomy chest radiography. Chest 1998; 113: 1647-9. [CrossRef]

17. Smith DK, Grillone GA, Fuleihan N. Use of postoperative chest $\mathrm{X}$-ray after elective adult tracheotomy. Otolaryngol Head Neck Surg 1999; 120: 848-51. [CrossRef]

18. Pappas S, Maragoudakis P, Vlastarakos P, Assimakopoulos D, Mandrali T, Kandiloros D, et al. Surgical versus percutaneous tracheostomy: an evidence-based approach. Eur Arch Otorhinolaryngol 2011; 268: 323-30. [CrossRef] 
19. De Leyn P, Bedert L, Delcroix M, Depuydt P, Lauwers G, Sokolov $\mathrm{Y}$, et al. Tracheotomy: clinical review and guidelines. Eur J Cardiothorac Surg 2007; 32: 412-21. [CrossRef]

20. Möller MG, Slaikeu JD, Bonelli P, Davis AT, Hoogeboom JE, Bonnell BW. Early tracheostomy versus late tracheostomy in the surgical intensive care unit. Am J Surg 2005; 189: 293-6. [CrossRef]

21. Flaatten H, Gjerde S, Heimdal JH, Aardal S. The effect of tracheostomy on outcome in intensive care unit patients. Acta Anaesthesiol Scand 2006; 50: 92-8. [CrossRef]

22. Shan L, Hao P, Xu F, Chen YG. Benefits of early tracheotomy: a meta-analysis based on 6 observational studies. Respir Care 2013; 58: 1856-62. [CrossRef]
23. Diaz-Prieto A, Mateu A, Gorriz M, Ortiga B, Truchero C, Sampietro $\mathrm{N}$, et al. A randomized clinical trial for the timing of tracheotomy in critically ill patients: factors precluding inclusion in a single center study. Crit Care 2014; 18: 585. [CrossRef]

24. Tong CC, Kleinberger AJ, Paolino J, Altman KW. Tracheotomy timing and outcomes in the critically ill. Otolaryngol Head Neck Surg 2012; 147: 44-51. [CrossRef]

25. Patel SA, Plowman EK, Halum S, Merati AL, Sardesai MG. Late tracheotomy is associated with higher morbidity and mortality in mechanically ventilated patients. Laryngoscope 2015; 125: 21348. [CrossRef] 